\title{
Study of elastic proton-proton single and double spin analyzing powers at RHIC HJET polarimeter
}

\author{
A. A. Poblaguev*† A. Zelenski, G. Atoian, E. Aschenauer, K.O. Eyser, H. Huang, \\ Y. Makdisi, and W. Schmidke \\ Brookhaven National Laboratory, Upton, NY 11973, USA \\ E-mail: poblaguev@bnl.gov
}

\section{Alekseev and D. Svirida}

Institute for Theoretical and Experimental Physics, Moscow 117218, Russia

\section{N. H. Buttimore}

Trinity College, University of Dublin, Ireland

\begin{abstract}
The experimental study of the single, $A_{\mathrm{N}}(t)$, and double, $A_{\mathrm{NN}}(t)$, spin analyzing powers was carried out at The Polarized Atomic Hydrogen Jet Target polarimeter (HJET) at The Relativistic Heavy Ion Collider (RHIC). Large statistics of elastic $p p$ events accumulated during RHIC Runs $2015\left(E_{\text {beam }}=100 \mathrm{GeV}\right)$ and $2017\left(E_{\text {beam }}=255 \mathrm{GeV}\right)$ allowed us to measure analyzing powers in the CNI region $0.001 \lesssim-t \lesssim 0.02 \mathrm{GeV}$ with unprecedented precision $\left|\delta A_{\mathrm{N}, \mathrm{NN}}\right| \lesssim 2 \times 10^{-4}$. The hadronic single spin-flip $r_{5}$ and double spin-flip $r_{2}$ amplitude parameters were reliably isolated. Based on this two beam energies measurement, Pomeron and Regge-pole contributions to the hadronic single- and double- spin-flip amplitudes were evaluated. Extrapolation of the measured $r_{5}(s)$ to $\sqrt{s}=200 \mathrm{GeV}$ is in agreement with STAR measurements at this energy. A possibility to use HJET data to search for an Odderon contribution to the spin-flip amplitudes is discussed.
\end{abstract}

PACS: $\quad 24.70 .+s, 25.40 . \mathrm{Cm}, 29.25 . \mathrm{Pj}, 29.85 . \mathrm{Fj}$

23rd International Spin Physics Symposium - SPIN2018 -

10-14 September, 2018

Ferrara, Italy

\footnotetext{
${ }^{*}$ Speaker.

${ }^{\dagger}$ Work supported by Brookhaven Science Associates, LLC under Contract No. DE-AC02-98CH10886 with the U.S. Department of Energy. Funding was also provided from the RIKEN BNL Research Center.
} 


\section{HJET Polarimeter at RHIC}

A precise measurement of the colliding beams polarization is an important component of the Relativistic Heavy Ion Collider (RHIC) Spin Program [1] at Brookhaven National Laboratory. The Polarized Atomic Hydrogen Gas Jet Target (HJET) [2] commissioned in 2004 was designed to measure absolute polarization of $24-255 \mathrm{GeV} / \mathrm{c}$ proton beams at RHIC with systematic errors better than $\Delta P / P \lesssim 0.05$. A significant upgrade of the polarimeter was done in 2015. It included the installation of new silicon detectors, increasing detector acceptance, and a new DAQ based on 250 MHz 12 bits WFD [3]. This, along with the development of new methods in data analysis, allowed us to reduce the systematic uncertainties of the beam polarization measurements to a $\sigma_{P}^{\text {syst }} / P \lesssim$ $0.5 \%$ level [4].

The HJET consists of three main components: an atomic beam source, a Breit-Rabi polarimeter to measure the atomic hydrogen polarization, and a recoil spectrometer to determine the beam and target (the jet) spin correlated asymmetries of the recoil proton angles. The recoil spectrometer is sketched in Fig. 1. The well defined vertical jet polarization, $P_{\text {jet }}=0.957 \pm 0.001$, is reversed every 5-10 minutes. Two, so-called blue and yellow, RHIC proton beams consist of alternating spin up/down bunches. At HJET, the polarizations of both beams are measured concurrently and continuously.

A detailed description of the data analysis methods used at the HJET was given in PSTP 2017 Proceedings [4]. It includes detector calibrations, identification of the backgrounds, isolation of elastic $p p$ events, and evaluation of the systematic uncertainties of the beam polarization measurements.

\section{Spin correlated asymmetries}

Using the HJET to measure proton beam polarization, the spin-correlated differential cross

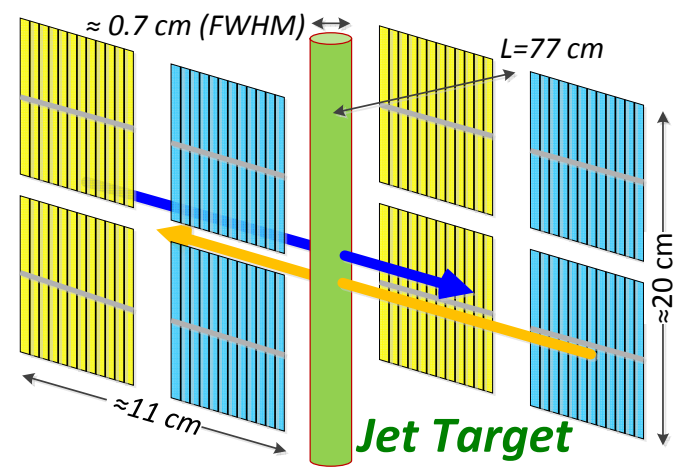

Figure 1: A schematic view of the HJET polarimeter. Eight silicon detectors, each with 12 readout channels, are optionally referred as blue and yellow depending on which RHIC beam they measure. 
section [5] dependence

$$
\frac{d^{2} \sigma}{d t d \varphi}=\frac{1}{2 \pi} \frac{d \sigma}{d t} \times\left[1+A_{\mathrm{N}} \sin \varphi\left(P_{j}+P_{b}\right)+\left(A_{\mathrm{NN}} \sin ^{2} \varphi+A_{\mathrm{SS}} \cos ^{2} \varphi\right) P_{b} P_{j}\right]
$$

on azimuth angle $\varphi$ are analyzed. Here, $P_{j}$ and $P_{b}$ are the jet and beam polarizations, respectively. Positive signs of the $P_{j, b}$ correspond to the spin up direction $(\varphi=0)$. The right/left (relative to a RHIC beam direction) detectors are located at $\sin \varphi= \pm 1$. Thus, the HJET measurements are sensitive only to analyzing powers $A_{\mathrm{N}}(s, t)$ and $A_{\mathrm{NN}}(s, t)$ which, generally, are functions of the center-of-mass energy squared $s=2 m_{p}\left(E_{\text {beam }}+m_{p}\right)$, and momentum transfer squared $-t=2 m_{p} T_{R}$.

The following spin correlated asymmetries can be experimentally determined (see Appendix A).

$$
a_{\mathrm{N}}^{\mathrm{j}}=\left\langle A_{\mathrm{N}}\right\rangle\left|P_{j}\right|, \quad a_{\mathrm{N}}^{\mathrm{b}}=\left\langle A_{\mathrm{N}}\right\rangle\left|P_{b}\right|, \quad a_{\mathrm{NN}}=\left\langle A_{\mathrm{NN}}\right\rangle\left|P_{j} P_{b}\right|
$$

where the measurement average values of the analyzing powers depend on the event selection cuts. For elastic $p p$ scattering, $\left\langle A_{\mathrm{N}}\right\rangle$ is the same for the jet and beam asymmetry which allows us to determine the beam polarization $\left|P_{b}\right|=\left(a_{\mathrm{N}}^{b} / a_{\mathrm{N}}^{j}\right)\left|P_{j}\right|$ with actually no knowledge of the analyzing power $A_{\mathrm{N}}(t)$. However, $A_{\mathrm{N}}(t)$ and $A_{\mathrm{NN}}(t)$ can be experimentally determined by measuring the dependence on the recoil proton kinetic energy $T_{R}$ of the asymmetries $a_{\mathrm{N}}\left(T_{R}\right)$ and $a_{\mathrm{NN}}\left(T_{R}\right)$.

Some systematic uncertainties which are effectively canceled in the beam polarization measurement have to be explicitly evaluated for the analyzing powers. These uncertainties include corrections to the background subtraction due to recoil proton tracking in the holding field magnet, to the effect of the finite vertical size of the detectors, and to the systematic uncertainties in the energy calibration.

For the Coulomb-nuclear interference (CNI) elastic $p p$ scattering at high energies, the theoretical basis for an experimental parametrization of the analyzing powers was given in Ref. [6] (BKLST). The analyzing powers

$$
\begin{aligned}
A_{\mathrm{N}}(t) & =\frac{\sqrt{-t}}{m_{p}} \frac{\left[\varkappa\left(1-\rho \delta_{C}+I_{2}\right)-2\left(I_{5}-\delta_{C} R_{5}\right)\right] \frac{t_{c}}{t}-2\left[\left(1+I_{2}\right) R_{5}-\left(\rho+R_{2}\right) I_{5}\right]}{\left(\frac{t_{c}}{t}\right)^{2}-2\left(\rho+\delta_{C}\right) \frac{t_{c}}{t}+1+\rho^{2}} \\
A_{\mathrm{NN}}(t) & =\frac{-2\left(R_{2}+\delta_{C} I_{2}\right) \frac{t_{c}}{t}+2\left(I_{2}+\rho R_{2}\right)-\left(\rho \varkappa-4 R_{5}\right) \frac{\varkappa t_{c}}{2 m_{p}^{2}}}{\left(\frac{t_{c}}{t}\right)^{2}-2\left(\rho+\delta_{C}\right) \frac{t_{c}}{t}+1+\rho^{2}}
\end{aligned}
$$

depend on the proton-proton forward $\operatorname{Re} / \operatorname{Im}$ ratio $\rho(s)$, total cross section $\sigma_{\mathrm{tot}}(s)=-8 \pi \alpha / t_{c}$, and slope $B(s)$ which can be extracted from unpolarized $p p$ data [7]. Here, $m_{p}$ is proton mass, $\varkappa=\mu_{p}-1=1.793$ is anomalous magnetic moment of proton. The Coulomb phase $\delta_{C}(t, B)$ and the hadronic single, $r_{5}=R_{5}+i I_{5}$, and double $r_{2}=R_{2}+i I_{2}$, spin-flip amplitude parameters are defined in Ref. [6]. For the values of $r_{2}$ measured in this work, the $r_{2}$ contribution to $A_{\mathrm{N}}$ can be neglected.

\section{Experimental determination of elastic $\boldsymbol{p}^{\uparrow} \boldsymbol{p}^{\uparrow}$ analyzing powers at the RHIC HJET}

In this work we analyze the HJET data acquired in two RHIC proton-proton runs: Run15 (100 $\mathrm{GeV}$ ) [8] and Run17 (255 GeV) [9]. About $2 \times 10^{9}$ elastic $p p$ events were selected at HJET in each 

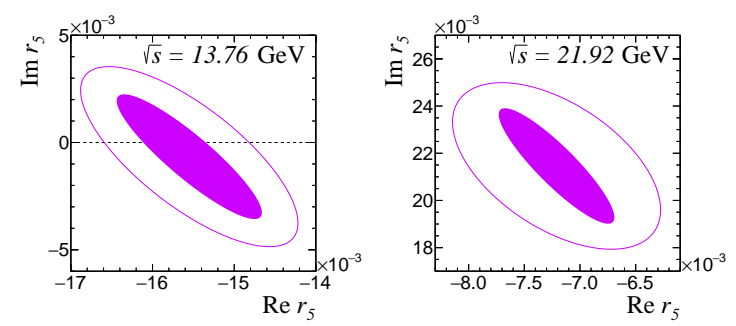

Figure 2: $1-\sigma$ correlation contours for the measured $r_{5}$. Solid lines correspond to the total (stat.+syst.) error while filled ellipses refer to statistical errors only.
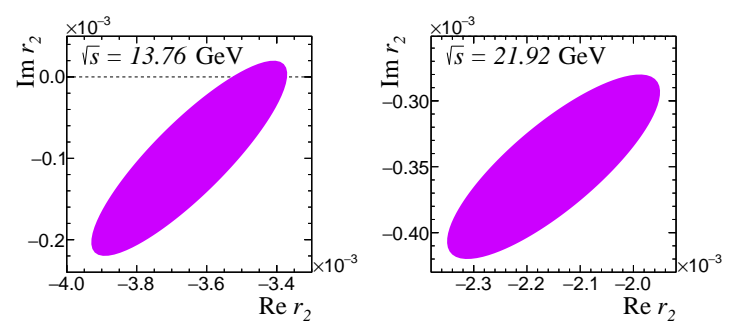

Figure 3: $1-\sigma$ correlation contours for the measured $r_{2}$. The experimental uncertainties are dominated by statistical errors.

Run. In the data analysis, the values of the total cross section $\sigma_{\text {tot }}$ and forward Re/Im ratio $\rho$ were taken from the $p p$ and $\bar{p} p$ data fit [10]. The slopes $B$ were derived from Ref. [11].

The Run specific conditions of the measurements can be briefly summarized as

Run15: $\sqrt{s}=13.76 \mathrm{GeV}, \rho=-0.079, \sigma_{\text {tot }}=38.39 \mathrm{mb}, B=11.2 \mathrm{GeV}^{-2}, P_{\text {jet }}^{\text {eff }}=0.954$.

Run17: $\sqrt{s}=21.92 \mathrm{GeV}, \rho=-0.009, \sigma_{\text {tot }}=39.19 \mathrm{mb}, B=11.6 \mathrm{GeV}^{-2}, P_{\text {jet }}^{\text {eff }}=0.953$.

$P_{\text {jet }}^{\text {eff }}$ is the effective jet polarization after systematic corrections.

To determine the hadronic spin-flip amplitude parameter $r_{5}$, we concurrently fit all four measured asymmetries $a_{\mathrm{N}}^{\mathrm{j}, \mathrm{b}}(t)=P_{\mathrm{j}, \mathrm{b}} A_{\mathrm{N}}\left(t, r_{5}\right)$. The polarizations of each of the blue and yellow beams were considered as free parameters in the fit. The results are shown in Fig. 2. One can see that $\left|r_{5}\right|$ is distinctly non-zero at both energies. Similarly, the result of measurement of $r_{2}$ is displayed in Fig. 3.

\section{Evaluation of the energy dependence of analyzing powers $A_{\mathrm{N}}(s, t)$ and $A_{\mathrm{NN}}(s, t)$}

For unpolarized protons, elastic $p p(\bar{p} p)$ scattering can be described accurately with five Regge poles, namely, Pomeron $P$ and the dominant $C= \pm 1$ poles for $I=0,1$, encoded by $R^{+}$for $\left(f_{2}, a_{2}\right)$ and $R^{-}$for $(\omega, \rho)$ [10]. In this approximation, the unpolarized amplitude may be presented as a sum of Regge pole contributions

$$
\sigma_{\mathrm{tot}}(s) \times[\rho(s)+i]=\sum_{R=P, R^{ \pm}}\left[R_{R}(s)+i I_{R}(s)\right]
$$

The real functions $R_{R}(s)$ and $I_{R}(s)$ have been determined in the unpolarized elastic $p p$ and $\bar{p} p$ global fit [10] and are shown in Fig. 4.

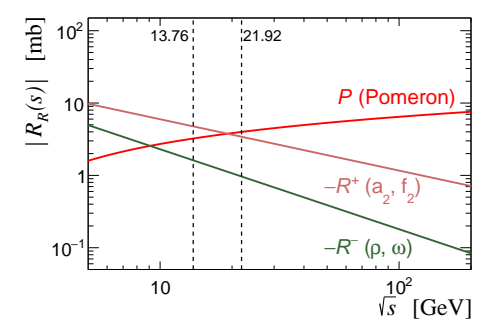

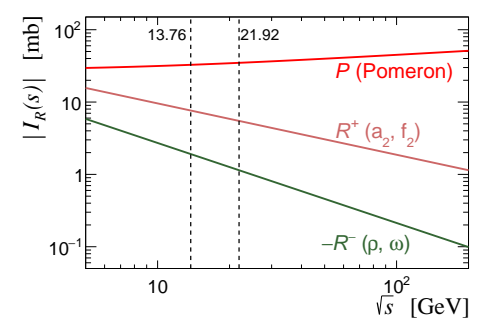

Figure 4: The Reggeon contributions to Eq. (4.1) obtained in the AU-L $\gamma=2(T)$ model of Ref. [10]. 

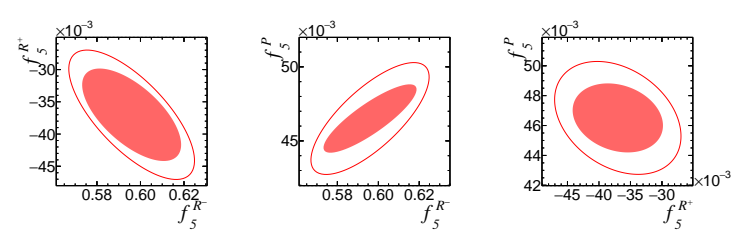

Figure 5: The Regeon single spin-flip couplings experimentally determined in this work.
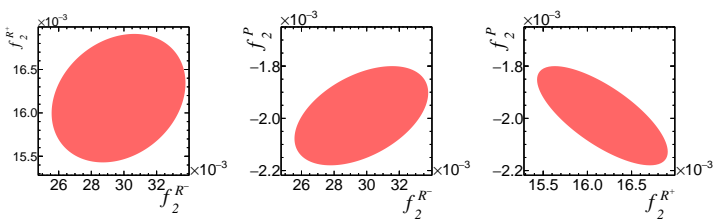

Figure 6: The Regeon double spin-flip couplings experimentally determined in this work.

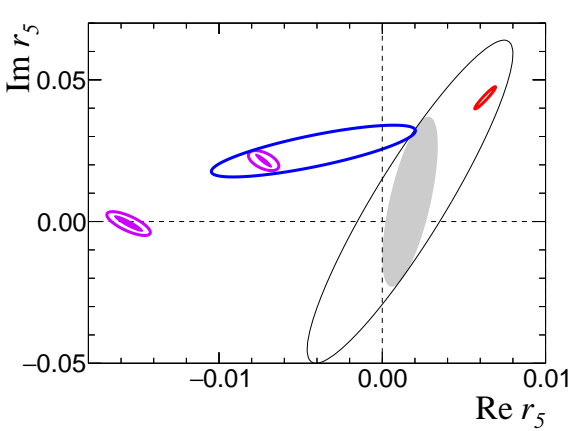

Figure 7: Extrapolation of the measured $r_{5}$ (violet color) to $\sqrt{s}=200 \mathrm{GeV}$ using the models of Fig. 4 (red) and Fig. 8 (blue). The STAR result [13] is shown by black (stat.+syst.) and grey (stat. only) colors.

Similarly, and again because they are analytic in $s$, the single and double spin-flip amplitudes can be expanded in terms of real coefficients, $f_{5,2}^{R}$, of the form [12]

$$
\sigma_{\mathrm{tot}}(s) \times r_{5,2}(s)=\sum_{R=P, R^{ \pm}} f_{5,2}^{R} \times\left[R_{R}(s)+i I_{R}(s)\right]
$$

where the constants, $f_{5,2}^{R}$, are single and double spin-flip to non-flip coupling ratios.

In the combined fit of the 100 and $255 \mathrm{GeV}$ HJET data, we determined the single (Fig. 5) and double (Fig. 6) spin-flip couplings. The Pomeron couplings are well identified in both cases.

Using the result of the fit we can extrapolate the value of $r_{5}$ to $\sqrt{s}=200 \mathrm{GeV}$ which allows us to compare the HJET and the STAR [13] measurements. An impressive consistency was observed (Fig. 7).

We also fit HJET hadronic spin-flip amplitudes using the Froissaron-Maximal-Odderon model of Ref. [14]. The parametrization (Fig. 8) was suggested to explain the surprisingly low value of
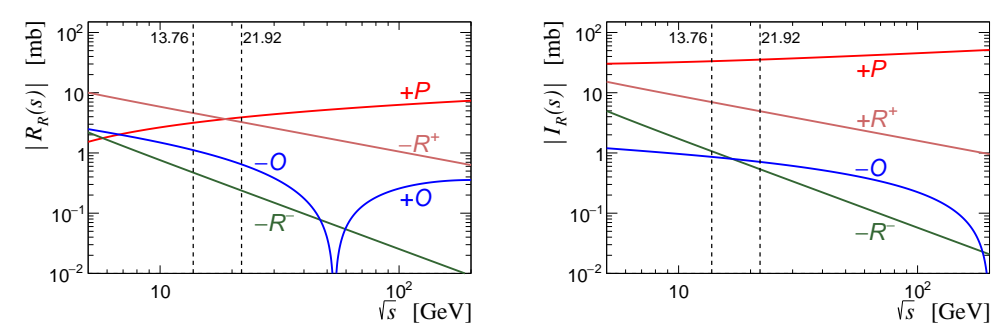

Figure 8: The Reggeon contributions including the Odderon one $\left(R_{O}+i I_{O}\right)$ in the FroissaronMaximal-Odderon model of Ref. [14].
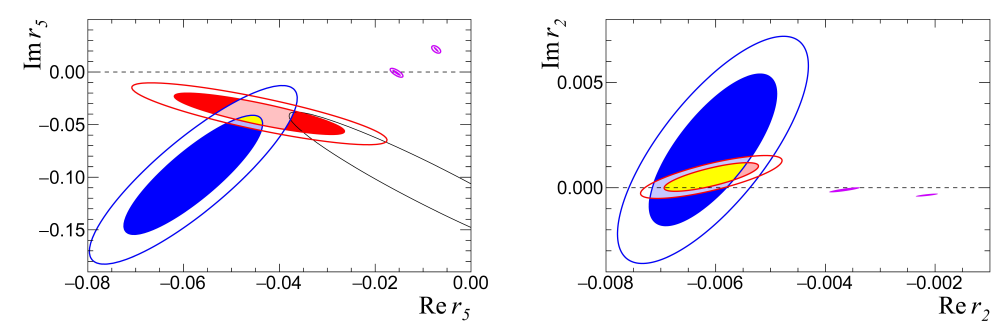

Figure 9: Extrapolation of the $r_{5}$ and $r_{2}$ to $\sqrt{s}=6.8 \mathrm{GeV} .2-\sigma$ and $3-\sigma$ correlation contours for the models of Fig. 4 (red) and Fig. 8 (blue) are shown. A violet color is used for this work and a black one for the earlier HJET [16] results. 

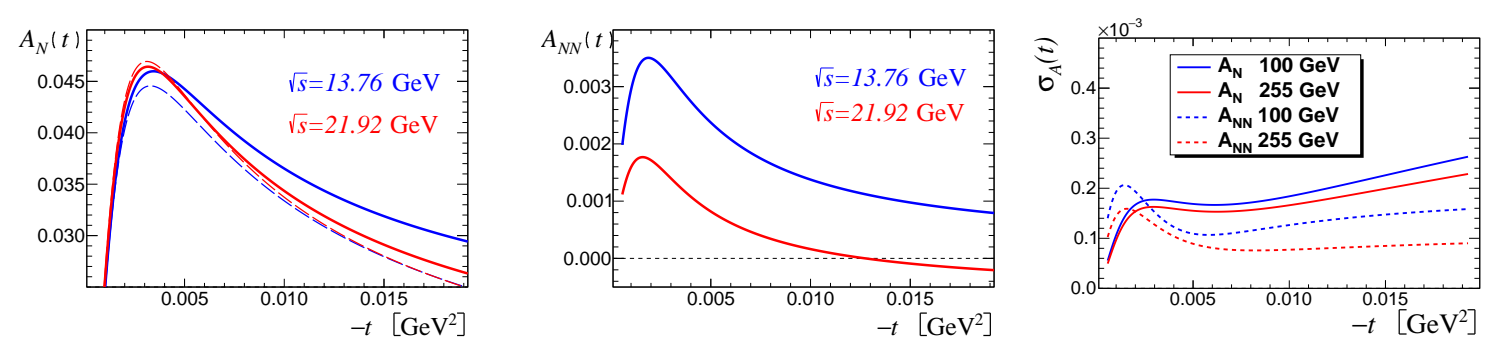

Figure 10: Elastic $p p$ analyzing powers $A_{\mathrm{N}}(t)$ and $A_{\mathrm{NN}}(t)$ measured in this work. For $A_{\mathrm{N}}(t)$, the dashed lines refer to the expected analyzing powers if $r_{5}=0$. The experimental uncertainties (stat.+syst.) are shown in the right graph.

$\rho=0.10 \pm 0.01$ at $\sqrt{s}=13 \mathrm{TeV}$ measured by the TOTEM experiment [15]. The $r_{5}$ extrapolation to $\sqrt{s}=200 \mathrm{GeV}$ is shown in Fig. 7. Since in the existing two point (100 and $255 \mathrm{GeV}$ proton beam energy) data the $R_{O}+i I_{O}$ can be well appoximated by a linear combinations of $R^{ \pm}$and $P$ functions, the result was uncertain. Nonetheless, it should be pointed out (Fig. 9) that an additional precision measurement at other beam energy, e.g. by HJET at RHIC injection, $24 \mathrm{GeV}$, might be a sensitive test of the hypothesis [14].

\section{Summary}

The HJET polarimeter at RHIC was used to study the spin-correlated asymmetries in elastic scattering of transversly polarized protons. The data acquired in RHIC Runs 2015 (100 GeV beam energy) and $2017(255 \mathrm{GeV})$ allowed us to determine single and double spin analyzing powers in the CNI region $0.0013<-t<0.018 \mathrm{GeV}$ with unprecedented precision as shown in Fig. 10.

The hadronic spin-flip amplitudes parameters $r_{5}$ and $r_{2}$ were reliably isolated at both energies. The measurements at two energies allowed us to evaluate the Regge pole expansion of the spin-flip amplitudes $\sigma_{\text {tot }}(s) r_{5}(s)$ and $\sigma_{\text {tot }}(s) r_{2}(s)$. Pomeron single and double spin-flip couplings were well determined. The $r_{5}$ extrapolation to $\sqrt{s}=200 \mathrm{GeV}$ is notably consistent with STAR measurements [13].

It was recently pointed out by Boris Kopeliovich [17] that the BKLST $A_{\mathrm{N}}(t)$ was derived with some simplifications, namely, $(i)$ the difference between electromagnetic and hadronic form factors was neglected and (ii) the absorptive correction on inelastic collisions was not considered. For the precision measurement at HJET, such corrections may be essential.

However, if the absorption can be approximated in the CNI region by a linear correction to the electromagnetic form factor $F^{\mathrm{em}}(t) \rightarrow F^{\mathrm{em}} \times(1+a t)$ then all such corrections can be accumulated in the values of $\operatorname{Re} r_{5}$ and $\operatorname{Im} r_{5}$. In other words, the measured analyzing power (Fig. 10) is not affected by the corrections but a biased value of $r_{5}$ is measured. Consequently, the Reggeon coupling fit may also depend on the corrections.

\section{Acknowledgments}

We thank the Collider Accelerator Department and the RHIC/AGS Operation Groups. We also would like to thank B. Z. Kopeliovich and M. Krelina for useful discussions. N. H. Buttimore is 
grateful for partial support from the Hamilton Mathematics Institute.

\section{A. Experimental determination of the spin correlated asymmetries}

At HJET, the jet/beam spin correlated asymmetries $a_{\mathrm{N}}^{\mathrm{j}}, a_{\mathrm{N}}^{\mathrm{b}}$, and $a_{\mathrm{NN}}$, the jet/beam intensity asymmetries $\lambda_{\mathrm{j}}$ and $\lambda_{\mathrm{b}}$, and the right/left detector acceptance asymmetry $\varepsilon$ can be derived from the selected elastic event counts discriminated by the right/left $(R L)$ detector location and the beam $(\uparrow \downarrow)$ and jet $(+-)$ spin directions:

$$
N_{R L}^{(\uparrow \downarrow)(+-)}=N_{0}\left(1+\eta_{\varepsilon} \eta_{j} a_{\mathrm{N}}^{\mathrm{j}}+\eta_{\varepsilon} \eta_{b} a_{\mathrm{N}}^{\mathrm{b}}+\eta_{j} \eta_{b} a_{\mathrm{NN}}\right)\left(1+\eta_{j} \lambda_{\mathrm{j}}\right)\left(1+\eta_{b} \lambda_{\mathrm{b}}\right)\left(1+\eta_{\varepsilon} \varepsilon\right)
$$

where the sign symbol $\eta_{j}(+-)= \pm 1$ is defined by the jet spin $(+-)$ and similarly for $\eta_{b}(\uparrow \downarrow)$ and $\eta_{\varepsilon}(R L)$. The Eqs. (A.1) can be solved analytically:

$$
\begin{aligned}
& a_{\mathrm{N}}^{\mathrm{j}}= \frac{\sqrt{N_{R}^{\uparrow+} N_{L}^{\downarrow-}}+\sqrt{N_{R}^{\downarrow+} N_{L}^{\uparrow-}}-\sqrt{N_{R}^{\uparrow-} N_{L}^{\downarrow+}}-\sqrt{N_{R}^{\downarrow-} N_{L}^{\uparrow+}}}{\sqrt{N_{R}^{\uparrow+} N_{L}^{\downarrow-}}+\sqrt{N_{R}^{\downarrow+} N_{L}^{\uparrow-}}+\sqrt{N_{R}^{\uparrow-} N_{L}^{\downarrow+}}+\sqrt{N_{R}^{\downarrow-} N_{L}^{\uparrow+}}} \\
& a_{\mathrm{N}}^{\mathrm{b}}= \frac{\sqrt{N_{R}^{\uparrow+} N_{L}^{\downarrow-}}-\sqrt{N_{R}^{\downarrow+} N_{L}^{\uparrow-}}+\sqrt{N_{R}^{\uparrow-} N_{L}^{\downarrow+}}-\sqrt{N_{R}^{\downarrow-} N_{L}^{\uparrow+}}}{\sqrt{N_{R}^{\uparrow+} N_{L}^{\downarrow-}}+\sqrt{N_{R}^{\downarrow+} N_{L}^{\uparrow-}}+\sqrt{N_{R}^{\uparrow-} N_{L}^{\downarrow+}}+\sqrt{N_{R}^{\downarrow-} N_{L}^{\uparrow+}}} \\
& a_{\mathrm{NN}}=\frac{\sqrt{N_{R}^{\uparrow+} N_{L}^{\downarrow-}}-\sqrt{N_{R}^{\downarrow+} N_{L}^{\uparrow-}}-\sqrt{N_{R}^{\uparrow-} N_{L}^{\downarrow+}}+\sqrt{N_{R}^{\downarrow-} N_{L}^{\uparrow+}}}{\sqrt{N_{R}^{\uparrow+} N_{L}^{\downarrow-}}+\sqrt{N_{R}^{\downarrow+}} N_{L}^{\uparrow-}+\sqrt{N_{R}^{\uparrow-} N_{L}^{\downarrow+}}+\sqrt{N_{R}^{\downarrow-} N_{L}^{\uparrow+}}} \\
& \lambda_{\mathrm{b}}=\frac{\sqrt[4]{N_{R}^{\uparrow+} N_{R}^{\downarrow+} N_{L}^{\uparrow+} N_{L}^{\downarrow+}}-\sqrt[4]{N_{R}^{\uparrow-} N_{R}^{\downarrow-} N_{L}^{\uparrow-} N_{L}^{\downarrow-}}}{\sqrt[4]{N_{R}^{\uparrow+} N_{R}^{\downarrow+} N_{L}^{\uparrow+} N_{L}^{\downarrow+}}+\sqrt[4]{N_{R}^{\uparrow-} N_{R}^{\downarrow-} N_{L}^{\uparrow-} N_{L}^{\downarrow-}}} \\
& \varepsilon=\frac{\sqrt[4]{N_{R}^{\uparrow+} N_{R}^{\uparrow-} N_{L}^{\uparrow+} N_{L}^{\uparrow-}}-\sqrt[4]{N_{R}^{\downarrow+} N_{R}^{\downarrow-} N_{L}^{\downarrow+} N_{L}^{\downarrow-}}}{\sqrt[4]{N_{R}^{\uparrow+} N_{R}^{\uparrow-} N_{L}^{\uparrow+} N_{L}^{\uparrow-}}+\sqrt[4]{N_{R}^{\downarrow+} N_{R}^{\downarrow-} N_{L}^{\downarrow+} N_{L}^{\downarrow-}}} \\
& b_{\mathrm{NN}}=\frac{\sqrt[4]{N_{R}^{\uparrow+} N_{R}^{\downarrow+} N_{R}^{\uparrow-} N_{R}^{\downarrow-}}-\sqrt[4]{N_{L}^{\uparrow+} N_{L}^{\downarrow+} N_{L}^{\uparrow-} N_{L}^{\downarrow-}}}{\sqrt[4]{N_{R}^{\uparrow+} N_{R}^{\downarrow+} N_{R}^{\uparrow-} N_{R}^{\downarrow-}}+\sqrt[4]{N_{L}^{\uparrow+} N_{R}^{\downarrow+} N_{L}^{\uparrow-} N_{L}^{\uparrow+} N_{L}^{\downarrow-}}-\sqrt[4]{N_{R}^{\uparrow-} N_{L}^{\uparrow-} N_{R}^{\downarrow+} N_{L}^{\uparrow-} N_{L}^{\downarrow+}}}
\end{aligned}
$$

The value of $b_{\mathrm{NN}}$ has to be identically equal to 0 and, thus, it may be used as an indicator of possible systematic uncertainties. If all asymmetries are small, then they are statistically uncorrelated and have the same statistical error as defined by the total statistics $\sigma_{\text {stat }}=1 / \sqrt{N_{\text {tot }}}$.

\section{References}

[1] E. Aschenauer et al., arXiv:1501.01220.

[2] A. Zelenski et al., Nucl. Instrum. Meth. A 536, 248 (2005). 
[3] A. Poblaguev, PoS PSTP 2015, 032 (2015).

[4] A. Poblaguev et al., PoS PSTP 2017, 022 (2018).

[5] E. Leader, Spin in Particle Physics, p. 119, Cambridge University Press, 2001.

[6] N. H. Buttimore, B. Z. Kopeliovich, E. Leader, J. Soffer and T. L. Trueman, Phys. Rev. D 59, 114010 (1999).

[7] M. Tanabashi et al. (Particle Data Group), Phys. Rev. D 98, 030001 (2018).

[8] V. Schoefer et al., doi:10.18429/JACoW-IPAC2015-TUPWI060

[9] V. Ranjbar et al., doi:10.18429/JACoW-IPAC2017-TUPVA050

[10] D. A. Fagundes, M. J. Menon and P. V. R. G. Silva, Int. J. Mod. Phys. A 32, no. 32, 1750184 (2017).

[11] V. Bartenev et al., Phys. Rev. Lett. 31, 1088 (1973); Phys. Rev. Lett. 31, 1367 (1973).

[12] T. L. Trueman, Phys. Rev. D 77, 054005 (2008).

[13] L. Adamczyk et al., Phys. Lett. B 719, 62 (2013).

[14] E. Martynov and B. Nicolescu, Phys. Lett. B 778, 414 (2018).

[15] G. Antchev et al. [TOTEM Collaboration], arXiv:1812.04732 [hep-ex].

[16] I. G. Alekseev et al., Phys. Rev. D 79, 094014 (2009).

[17] B. Z. Kopeliovich and M. Krelina, these Proceedings. 